\title{
Power Control and Sub Carrier Grouping for MCCDMA-MIMO Using Water Filling Game Theory
}

\author{
A. Sundhar and P. Dananjayan
}

\begin{abstract}
The capacity enhancement in Multi Carrier Code Division Multiple Access (MC-CDMA) technique with multiple antennas at both the transmitter and the receiver is achieved by restricting interference noise through power control method at transmitter. Due to time varying nature of the channel, channel fading is not identical to all sub carriers. So, the allocation of sub carriers to the users according to the instantaneous Channel State Information (CSI) is also improves the capacity of the MCCDMA system. In this paper, The maximization of capacity in MCCDMA-MIMO system is achieved through dynamic allocation of sub carriers to each user and power allocation using water filling game theory subject to the constrain of total power at transmitter. The water filling algorithm distributes power among all users with the help of SINR that is received by the transmitter instead of getting full CSI. The sub carrier group assignment is implemented by selecting best sub carriers with maximum Signal to Interference and Noise Ratio (SINR) value and user's rate requirements. The performances of capacity improvements and outage probability reduction in MCCDMA-MIMO are analyzed with various diversity sizes (SISO, $2 \times 2,4 \times 4$ ).
\end{abstract}

Index Terms-CSI-Channel State Information, ICI-Inter Carrier Interference, IWFA- Iterative Water Filling Game Theoretic Approach, QoS- Quality of Services, MAIMultiple-Access Interference, MCCDMA- Multi Carrier Code Division Multiple access, MAI-Multiple Access Interference, SINR -Signal to Interference plus Noise Ratio.

\section{INTRODUCTION}

The application of multicarrier CDMA and multiple input-multiple output (MIMO) techniques in high data rate wireless communications over fading channels has become increasingly popular in recent years [1]. In comparison with single-carrier transmission, the key advantages of multicarrier transmission are the robustness against frequency-selective fading, narrowband interference and inter symbol interference (ISI). MIMO techniques employing multiple antennas at both the transmitter and receiver can provide robustness to space-selective fading and improved capacity. Due to the simultaneous transmissions, sub-carriers interfere with each other and limit the network performance. To achieve robustness and spectral efficiency in MC-CDMA system over multipath fading channels, the technology is ruled by two main functions; power allocation [2] over the sub carriers and sub carrier group assignment [3]. Power allocation is the scheme by which users share the power available at the serving base station. Group assignment

Manuscript received April 10, 2013; revised July 14, 2013

The authors are with the Department of ECE, Pondicherry Engineering College, Puducherry, India. (e-mail: aisundhar@rediffmail.com, pdananjayan@pec.edu). depicts selection of appropriate subcarriers to support information bit streams of individual users. So, the distributions of users across subcarrier groups as well as their transmission power distribution has a significant effect on QoS enhancement like mean capacity of the system and outage probability reduction.

The architecture of MCCDMA-MIMO system considered in this paper subdivides subcarriers into a set of non-overlapping subcarrier groups. A user in the system is assigned to the subcarrier group that holds the best CSI and transmitting the whole data of the user through that selected group of sub-carriers or varying the number of sub-carriers according to user's requirement. However, such performance gain comes at the expense of significant signaling overhead due to the sharing of channel state information (CSI) and transmission data. The CSI is subjected to the errors because of the imperfect channel estimation/measurement due to the time varying nature of the channels. So, this paper considered the Signal to- Interference/Noise Ratio (SINR) of the given user assigned on the subcarrier group as an objective function to distribute power along the sub carrier groups to restrict the interference noise.

The Game theory is an effective tool used to allocate power for each user with the independent knowledge of actual channel realization and gives solution for overall capacity maximization problem [4]. In the power control game, each user seeks to choose its transmitting power over each carrier to maximize its capacity. The water-filling rule in game theoretic perspective is a tool used to allocate proper power for every user in order to improve system capacity performance with global constraint (Total available power at base station) and imperfect CSI [5]. In this paper, the protocol to enhance the system capacity and outage probability by adopting the Iterative Water Filling Game theoretic Algorithm (IWGA) is implemented in MCCDMA-MIMO system [6], [7] and performances are analyzed with the threshold parameters SINR and Data rate.

The organization of this paper is as follows. Section II deals with the system architecture and game model for power control algorithm in MCCDMA -MIMO, strategy for power control, utilities function and problem formation to maximize the capacity. Section II describes solution for the problem and to maximize the payoff using water filling game theory. The results are depicted in Section IV and the conclusions are given in Section V.

\section{SyStem ARChiteCtURE AND GAME MODEL}

\section{A. System Architecture}

A multi cellular MC-CDMA network constituted of $\mathrm{B}=$ 
$\left\{1,2, \ldots, \mathrm{N}_{\mathrm{B}}\right\}$ base stations is considered. The system bandwidth ' $\mathrm{W}$ ' is subdivided into ' $\mathrm{N}_{\mathrm{C}}$ ' subcarriers. Bandwidth of subcarriers is selected such that they approximately exhibit flat fading channel characteristics (i.e., $W / N_{C} \leq B_{C}$, where ' $B_{C}$ ' is the coherence bandwidth). Each ' $G$ ' subcarriers constitute a group over which individual streams will be spread. As a result of subcarrier grouping, system bandwidth could be described in terms of a set of subcarrier groups, $\mathrm{C}=\left\{\mathrm{C}_{(1)}, \mathrm{C}_{(2)}, \ldots, \mathrm{C}_{(\mathrm{j})}, \ldots, \mathrm{C}_{(\mathrm{G})}\right\}$, where $N_{G}=N_{C} / G$ is the number of subcarrier groups. Each base station, $\mathrm{b} \in \mathrm{B}$, is effectively supporting ' $\mathrm{U}$ ' active data users. Each base station operates under the constraint that it has at its disposal a maximum amount of power to share among all active sessions. The symbol of user ' $u$ ' in the base station ' $b$ ' of an MCDMA system is presented as

$$
Y^{u}=H^{u} S^{u} \sqrt{P^{u}} X^{u}
$$

where

$X^{u}$ is the active user's symbol.

$S^{u}$ is the code matrix.

$P^{u}=\operatorname{diag}\left(P^{u 1}, P^{u 2}, P^{u 3}, \ldots, P^{U}\right)$ is each user's transmit power

$$
\begin{aligned}
& H^{u}=\operatorname{diag}\left(H^{u 1}, H^{u 2}, H^{u 3}, \ldots, H^{U}\right) \text { is each user's channel } \\
& \text { fading } \\
& Y^{u} \text { is MCCDMA Symbol Vector. }
\end{aligned}
$$

The time domain data stream for each user is divided into multiple parallel streams and each stream is spread using a spreading sequence as shown in Fig. 1.

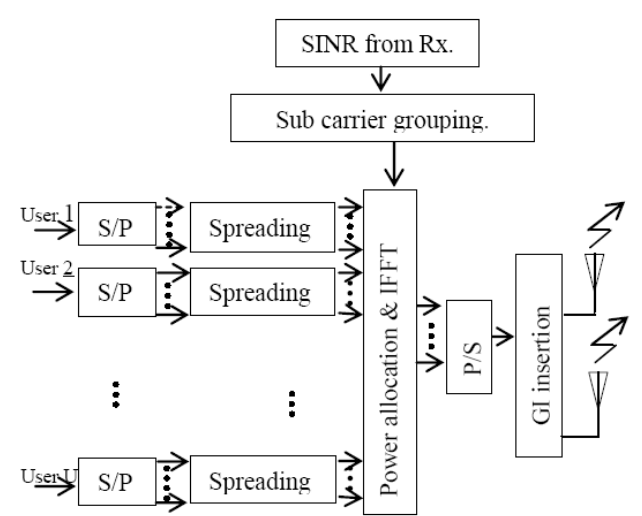

Fig. 1. MCCDMA-MIMO transmitter

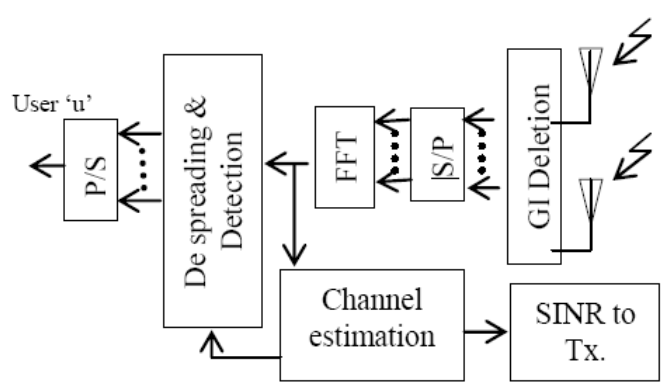

Fig. 2. MCCDMA-MIMO receiver

The sequences are then spread with sub carrier group domain using IFFT and power allocation to each user is followed by IWFA. The sub carrier group assignment is processed by CSI. The IWFA allocate power to each user with the help of SINR value as an objective function. The MC-CDMA symbols are then transmitted through MIMO structure of ' $\mathrm{N}_{\mathrm{t}}$ ' transmit antennas and ' $\mathrm{N}_{\mathrm{r}}$ ' receive antennas and is illustrated in Fig. 1 and Fig. 2 [8], [9].

\section{B. Game Model}

The game model in the MCCDMA transmitter plays against the behavior of a wireless channel and assigns power level to the respective sub carriers. The game model also considered that the CSI errors are uncorrelated across subcarriers. The strategies of this game are the possible power level which can be assigned on the sub carriers subject to the constraint of total power at the transmitter and total sub carriers. The objective function in the strategy of the game is the SINR value received from the receiver. A mobile user ' $u$ ' of interest served by base station ' $b$ ' has the SINR value given below.

$$
\operatorname{SINR}_{u}=\frac{\sum_{n=1}^{N_{c}} P_{n}^{u}\left|H_{n}^{u}\right|}{\sum_{u^{\prime}=1 ; u^{\prime} \neq u}^{U} P^{u^{\prime}}\left|H_{n}^{u^{\prime}}\right|+\left[E_{b^{\prime}}^{u}\right]}
$$

where

$\left[p^{U}\right]_{b}$ is power of user ' $\mathrm{u}$ ' in base station ' $\mathrm{b}$ '

$\left[I^{u}\right]_{b}$ is the interference to the user ' $u$ ' due to other users in the base station ' $b$ '.

$\left[E_{b^{\prime}}^{u}\right]$ is inter cell interference

This SINR value is considered as an objective function for the power control game to allocate the optimal power to each user. The channel capacity of the individual user ' $u$ ' in the base station ' $b$ ' is considered as utility function of the game and expressed as

$$
C^{u}=\sum_{n=1}^{n=N_{c}} \log _{2}\left(1+\frac{P_{n}^{u}}{N_{o} \sigma^{2}}\left|H_{n}^{u}\right|^{2}\right) \text { bits / s / Hz }
$$

The total system capacity of the base station ' $b$ ' that constitutes ' $\mathrm{U}$ ' active users[7] is given as

$$
C_{\text {Total }}=\sum_{n=1}^{n=U} C^{u}=\sum_{n=1}^{n=U} \sum_{n=1}^{n=N_{c}} \log _{2}\left(1+\frac{P_{n}^{u}}{N_{0} \sigma^{2}}\left|H_{n}^{u}\right|^{2}\right) \text { bits / s / Hz }
$$

Then the MCCDMA symbols are transmitted through MIMO system of $N_{t}, N_{r}$ antenna with channel matrix $H(\tau, t)$ [8], [9] as shown in Fig. 1 and system capacity or the utility function is modified as

$$
C_{\text {MCCDMA-MIMO }}=\log _{2}\left(\operatorname{det}\left[I_{N_{r}}+\frac{P^{u}}{N_{t} N_{r}}\left\{\frac{1}{N_{c}} \sum_{n=0}^{n=N_{c}} H(n) H^{+}(n)\right\}\right]\right) b i t s / s / H z
$$

\section{WATER FILling GAME THEORETIC SOLUTION}

The water filling game theory concept is modeled with selfish users and each user is interested in maximizing their own normalized effective capacity, or achievable throughput (rate), subject to an average power constraint [10], [11]. The 
capacity maximization of users in the MCCDMA-MIMO system with power constraint under Gaussian interference channel is optimized by sub carrier group forming and power distribution algorithm using water filling game theory. In order to maximize the capacity of the system, the power control game must calculate all utilities and expected payoffs for each one of his possible strategies.

To maximize the overall link capacity, users need to be grouped optimally under the interference and QoS requirements constraint, the optimization of the problem with respect to sub carrier and power constraint is formulated as

$$
\max \left(C^{u}\right)=\max \left[\sum_{i=1}^{G} \omega_{u}^{i} \sum_{u=1}^{U} \sum_{n=1}^{n=N_{c}} \log _{2}\left(1+\frac{P_{n}^{u}}{N_{0} \sigma^{2}}\left|H_{n}^{u}\right|^{2}\right)\right]
$$

Subject to $\quad \sum_{u=1}^{n=U} \sum_{n=1}^{n=N_{c}} P_{n}^{u} \leq P_{\text {Total }} \quad \sum_{i=1}^{i=G} \sum_{u=1}^{u=U} \omega_{u}^{i} \leq U$

where $\omega_{u}^{i} \in\{0,1\}$ is the allocation index for the active user ' $u$ ' in group ' $i$ '. That is, if $\omega_{u}^{i}=1$, then the user ' $u$ ' is assigned to the group ' $i$ '. if $\omega_{u}^{i}=0$, then the user ' $u$ ' is not assigned to the group ' $i$ '. The solution for capacity maximization problem of single-water level, multi-constraint (Power and group selection constraint) is obtained through iterative method by simple fixing the water level ' $\mu$ ' (power level) and adjusting it iteratively until the constraint is satisfied.The power level of user ' $u$ ' in the base station ' $b$ ' is obtained using Lagrange multipliers as given as

$$
\begin{gathered}
P_{A^{*}}^{u^{*}}=\left[\frac{1}{U}\left(P_{\text {Total }}-\sum_{n-1}^{U} \sum_{n=1}^{N_{c}} \frac{\sigma^{2}}{\left|H_{n}^{u}\right|^{2}}\right)-\frac{\sigma^{2}}{\left|H_{n^{*}}^{u^{*}}\right|^{2}}\right]^{+} \\
{[x]^{+}=\max \{x, 0\}}
\end{gathered}
$$

Here the power level and group selection of each user are obtained simply by fixing the water level (power level) $P_{A^{*}}^{u^{*}}$, QoS requirement of individual user and then adjusting it iteratively until the constraints are satisfied as per the algorithm given below[12], [13].

\section{Algorithm:}

Step 1: Initialization

$N_{c} \rightarrow$ Number of subcarriers

$U \rightarrow$ Total number of users

$G \rightarrow$ No of groups

$P_{t} \rightarrow$ Total Power budget

Step 2: Get $\mathrm{SINR}_{\mathrm{u}} \rightarrow$ SINRfor user ' $\mathrm{u}$ '

Get QoS Requirements for user ' $u$ '

Step 3: Group Assignment and Power Allocation ( without water filling algorithm)

Calculate $\lambda=H_{n}^{u} \rightarrow$ channel gain of each user groups
Compute average SINR value for all groups and arrange in order

Arrange the user priority according to the QoS requirements

Assign the groups to the user according to the order

Calculate $P_{A^{*}}^{u^{*}} \&$ assign the same to user ' $\mathrm{u}$ '

Step 4: Power Allocation (IWFA)

Calculate $\lambda=H_{n}^{u} \rightarrow$ channel gain of each user groups

Compute average SINR value for all groups and arrange in order

$$
\text { Fix: } \mu=P_{A^{*}}^{u^{*}} \& \quad \lambda=H_{n}^{u}
$$

Loop 1: Number of iterations

Fix step size(0.001)

Loop 2: Group assignment

$$
\begin{aligned}
& P_{A}^{u}(\mathrm{i})=P_{A}^{u}(\mathrm{i})+\text { step size } \\
& \text { Check } \\
& \text { Power Constraint : } \sum_{u=1}^{U} \sum_{k=1}^{N_{c}} P_{k}^{u} \leq P_{\text {Total }} \\
& \text { Group Constraint : } \sum_{i=1}^{i=G} \sum_{u=1}^{u=U} \omega_{u}^{i} \leq U \\
& \text { Capacity Constraint: } C_{u}^{i} \geq \text { Data rate }
\end{aligned}
$$

If satisfied

$$
P_{k}^{u}(i)=P_{k}^{u}(i)+\text { stepsize }
$$

Else

$$
\begin{aligned}
& \text { Assign } \\
& P_{k}^{u}(i)=P_{k}^{u}(i)
\end{aligned}
$$

User ' $u$ ' is assigned to group ' $i$ '

Capacity of the user $=C_{u}^{i}$

End loop 2

End loop 1

Step 5 : End

\section{RESUlts AND ANALYSIS}

The capacity improvements and outage probability of MCCDMA are analyzed with the numerical conditions summarized in Table 1 and platform used for the simulation is MATLAB 7.0. The power allocation scheme to enhance the capacity is optimized by IWFA in the presence of imperfect CSI. The IWFA takes 1000 iterations to allocate power to each user using 10000 Monte Carlo Channel realizations.

The group assignment strategy to maximize the overall mean capacity using IWFA is followed by the algorithm mentioned in section III. The capacity improvement of MCCDMA in SISO using IWFA is compared with power allocation without water filling algorithm. The Further capacity enhancement of MCCDMA through MIMO multiplexing $(2 \times 2,4 \times 4)$ is also analyzed with and without IWFA. Fig. 3 shows the capacity improvement analysis of MCCDMA-MIMO (SISO, 2×2, 4×4) using IWFA with interference based sub carrier grouping. The MCCDMA-MIMO system with water filling has a greater 
mean capacity as compared to the system without water filling.

TABLE I: SIMULATION PARAMETERS

\begin{tabular}{|l|l|}
\hline \multicolumn{1}{|c|}{ Parameters } & \multicolumn{1}{c|}{ Specifications } \\
\hline MCCDMA system & Multi cellular structure with three cell \\
\hline No of carriers $\left(\mathrm{N}_{\mathrm{c}}\right)$ & 1024 \\
\hline Spreading factors & 1024 \\
\hline Multipath channel model & Rayleigh fading \\
\hline Channel condition & AWGN channel \\
\hline Modulation & QAM \\
\hline MIMO sizes & $2 \times 2$ and $4 \times 4$ \\
\hline $\begin{array}{l}\text { Monte Carlo Channel } \\
\text { realization }\end{array}$ & 10000 \\
\hline Number of iterations & 1000 \\
\hline SINR range & -10 dB to30dB \\
\hline Detector & Zero forcing detector \\
\hline Eb/No range & 0 to 25 dB \\
\hline
\end{tabular}

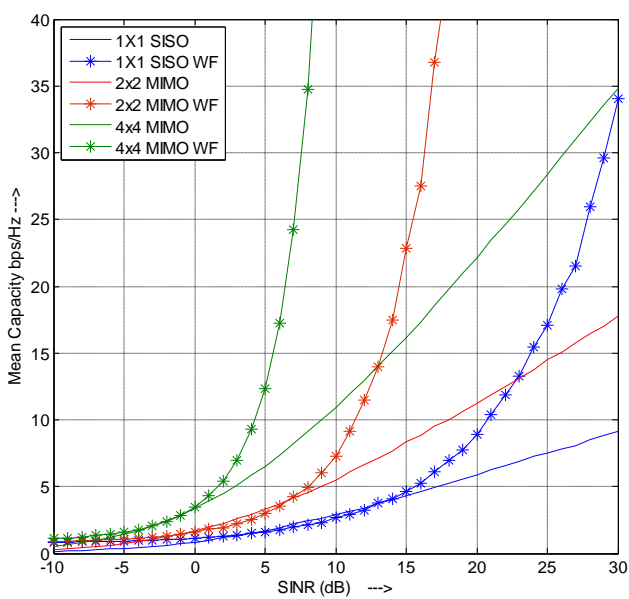

Fig. 3. Mean capacity vs sinr of MCCDMA-MIMO with sub carrier group assignment using iwfa based power allocation.

The capacity enhancement of the system is due to the proper power allocation to all users by IWFA with sub carrier group assignment strategy. The algorithm limits the interference noise among all users by avoiding excess power to any particular user and assigns the compatible sub carrier group according to data rate demands. The signal transmission of the system through MIMO gives an additional improvement of the system capacity.

In MC-CDMA systems, although there is no hard limit on the number of concurrent users, there is a practical limit to control the interference between users. Otherwise, the QoS requirements of the system cannot be guaranteed. So, the system capacity is determined under the condition that the outage probability does not exceed the required threshold. The general outage probability formula is given by

$$
\begin{gathered}
p(\text { outage })=1-p\left(\operatorname{SINR}<\operatorname{SINR}_{\text {threshold }}\right) \\
p(\text { outage })=1-p\left(C<C_{\operatorname{Re} q}\right)
\end{gathered}
$$

This outage probability parameter is a key figure of merit of system and can be calculated with the help of probability distribution [14] of all Monte Carlo signals involved in the fluctuations. The minimization of the outage probability is achieved through intelligent way of determining transmission power of each user using IWFA. The performances of outage probability (Probability that the interference noise power of Monte Carlo Channel exceeds the signal power) with respect to SINR value range from 2 to $20 \mathrm{~dB}$ for the MCCDMA-MIMO system using IWFA is depicted in Fig. 4. The performance of outage probability (Probability that the actual data rate of the system falls below the expected data rate of the system) is also analyzed and depicted in Fig. 5.

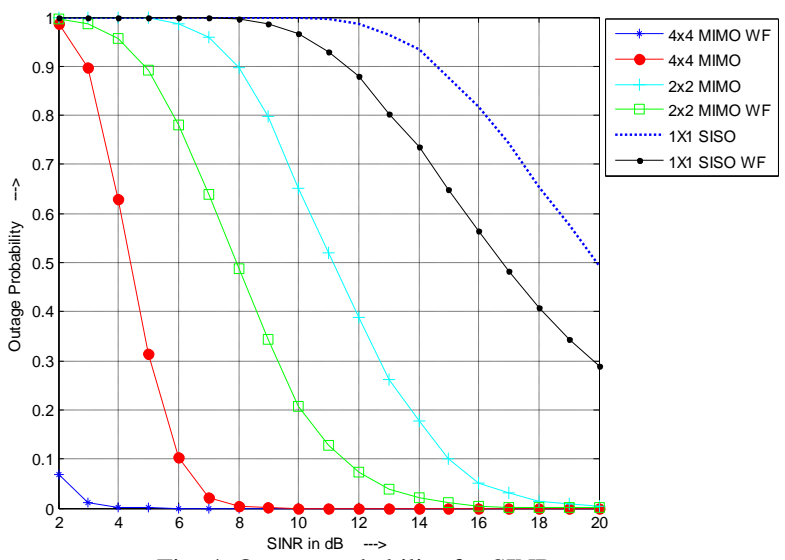

Fig. 4. Outage probability for SINR range

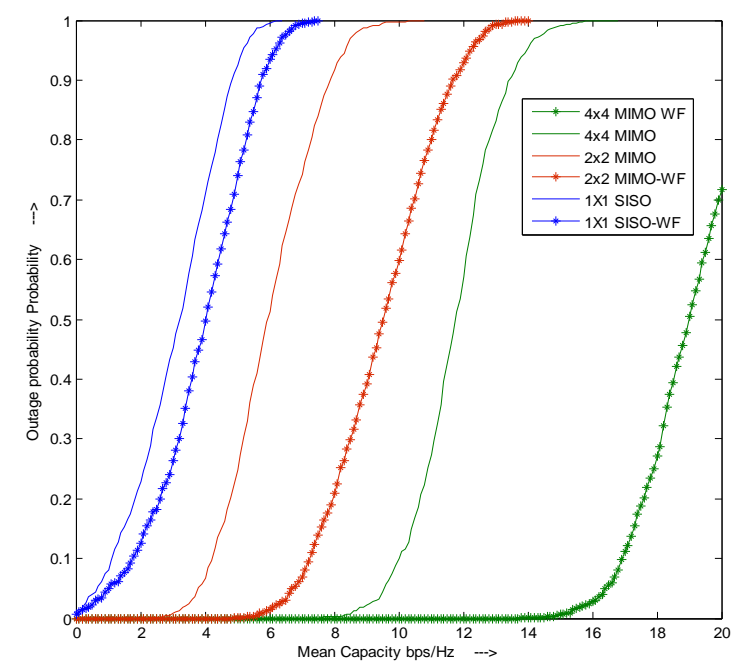

Fig. 5. Outage probability for capacity range

\section{CONCLUSION}

The capacity enhancement and outage probability reduction in MCDMA is achieved by IWFA based power control and sub carrier group assignment method. The power distribution to each user using IWFA is modeled based on the SINR value as an objective function which is received from receiver in the presence of imperfect CSI. This paper demonstrated that the interference based sub carrier group assignment strategy with power control through IWFA enhance the capacity and outage probability of the system by restricting interference noise. The further improvements of capacity and outage probability in MCCDMA are achieved by exploiting MIMO diversity of size $2 \times 2$ and $4 \times 4$. The mean capacity improvements and reduction of outage probability in MCCDMA-MIMO system using IWFA are analyzed. The capacity in MCCDMA is enhanced nearly $10 \%$ for SISO and $32 \%$ for MIMO due to power allocation using Iterative Water Filling game theoretic Approach. The IWFA based power 
control algorithm also benefits the MCCDMA-MIMO system by minimizing the overall outage probability of the system.

\section{REFERENCES}

[1] S. L. Nours, F. Nouvel, and J. F. Helard, "Design and Implementation of MC-CDMA systems for future wireless networks," EURASIP Journal on Applied Signal Processing, vol. 2004, pp. 1604-1615, January 2004.

[2] Y. H. Lee and Y. Bar-Ness, "Transmission power adaptations in mc-cdmacommunications over rayleigh fading channels," IEEE transactions on VehicularTechnology, vol. 56, no. 6, pp. 3486-3494, November 2007.

[3] T. El Shabrawy and T. Le-Ngoc, "Subcarrier group assignment for MC-CDMA wireless networks," EURASIP Journal on Wireless Communications and Networking, 2007.

[4] F. Meshkati, M. Chiang, H. V. Poor, and S. C. Schwartz, "A game-theoretic approach to energy-efficient power control in multicarrier cdma systems," IEEE Journal on Selected Areas in Communications, vol. 24, no. 6, June 2006.

[5] D. P. Palomar and J. R. Fonollosa, "Practical algorithm for a family of water-filling solutions," IEEE transactions on Signal Processing, vol. 53, no. 2, pp. 686-695, February 2005.

[6] A. Sundhar and P. Dananjayan, "Capacity Enhancement in MCCDMA-MIMO System Using Iterative Water Filling Approach Based Power Distribution Method," in Proc. IEEE International Conference on Computing Communication and Networking Technologies, Coimbatore, India, pp. 1-5, July 2012.

[7] D. K. Kim and D. K. Sung, "Capacity estimation for an SIR-based power-controlled CDMA system supporting ON-OFF traffic," IEEE transactions on Vehicular Technology, vol. 49, no. 4, pp. 1094-1101, July 2000.

[8] S. Catreux, P. F. Driessen, and L. J. Greenstein, "Attainable throughput of an interference- limited multiple-input multiple-output cellular system," IEEE Transactions on communications, vol. 49, no. 8, pp. 1307-1311, August 2001.

[9] Y. Rong and Y. B. Hua, "Optimal power schedule for distributed MIMO links," IEEE Transactions on Wireless communications, vol. 7, no. 8, pp. 2896-2900, August 2008.

[10] Y. C. Wang, P. Zhang, Y. A. Liu, and N. Li, "Suboptimal resource allocation with MMSE detector for grouped MC-CDMA systems," The Journal of China Universities of Posts and Telecommunications, vol. 15, no. 3, pp. 30- 36, September 2008.

[11] A. Sundhar and P. Dananjayan, "Interference based sub carrier group assignment and power distribution for MCCDMA-MIMO system using iterative water filling game theory," in Proc. IASTED International
Conference on Engineering and Applied Science, Sri Lanka, Dec. 2012.

[12] K. Adachi, F. Adachi, and M. Nakagawa, "A Study on Channel Capacities of MC-CDMAMIMO and OFDMMIMO," in Proc. IEEE International Conference on Communication Systems, Singapore, pp. 1384-1388, November 2008.

[13] E. S. Lo, P. W. C. Chan, V. K. N. Lau, R. S. Cheng, K. B. Letaief, R. Murch, and W. H. Mow, "Adaptive Resource Allocation and Capacity Comparison of Downlink Multiuser MIMO-MC-CDMA and MIMO-OFDMA," IEEE Transactions on Wireless Communications, vol. 6, no. 3, pp. 1083-1093, March 2007.

[14] L. F Li and A. J. Goldsmith, "Capacity and Optimal Resource Allocation for FadingBroadcast Channels-Part II: Outage Capacity," IEEE Transactions on Information Theory, vol. 47, no. 3, March 2001.

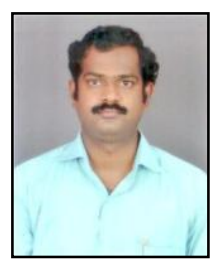

A. Sundhar received B.Tech degree in Electronics and Communication Engineering from Pondicherry University, Pondicherry, India in 2002 and M.Tech degree in Electronics and Communication Engineering from Pondicherry Engineering College, Pondicherry in 2005. He is serving as Assistant Professor in Department of Electronics and Communication Engineering, Perunthalaivar Kamarajar Institute of Engineering and technology, Karaikal, India. He is pursuing his Ph.D. in the Department of Electronics and Communication Engineering, Pondicherry Engineering College, Pondicherry. His research interests include wireless mobile communication, multi carrier communication and MIMO.

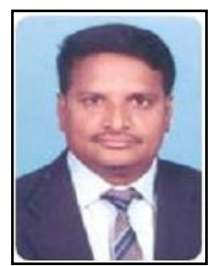

P. Dananjayan received Bachelor of Science from University of Madras in 1979, Bachelor of Technology in 1982 and Master of Engineering in 1984 from the Madras Institute of Technology, Chennai and Ph.D. degree from Anna University, Chennai in 1998. He is working as Professor in the Department of Electronics and Communication Engineering and Chairman (PG programs), Pondicherry Engineering College, Pondicherry, India. He has been as visiting professor to AIT, Bangkok. He has to his credit more than 90 publications in National and International Journals. He has presented more than 160 papers in National and International Conferences. He has guided $12 \mathrm{Ph} . \mathrm{D}$ candidates and is currently guiding $7 \mathrm{Ph} . \mathrm{D}$ students. His research interests include spread spectrum techniques, wireless communication, and wireless adhoc and sensor networks. 